\title{
Quantitative histology-based classification system for assessment of the intestinal mucosal histological changes in patients with celiac disease
}

\author{
Prasenjit Das ${ }^{1}$, Gaurav PS Gahlot ${ }^{1}$, Alka Singh ${ }^{2}$, Vandana Baloda ${ }^{1}$, Ramakant Rawat ${ }^{2}$, Anil K Verma ${ }^{2 \S}$, \\ Gaurav Khanna ${ }^{1}$, Maitrayee Roy ${ }^{1}$, Archana George ${ }^{1}$, Ashok Singh ${ }^{1}$, Aasma Nalwa ${ }^{1}$, Prashant Ramteke ${ }^{1}$, Rajni Yadav ${ }^{1}$, \\ Vineet Ahuja ${ }^{2}$, Vishnubhatla Sreenivas ${ }^{3}$, Siddhartha Datta Gupta ${ }^{1}$, Govind K Makharia ${ }^{2}$ \\ Departments of ${ }^{1}$ Pathology, ${ }^{2}$ Gastroenterology and Human Nutrition, and ${ }^{3}$ Biostatistics, All India Institute of Medical Sciences, New Delhi, India
}

Background/Aims: The existing histological classifications for the interpretation of small intestinal biopsies are based on qualitative parameters with high intraobserver and interobserver variations. We have developed and propose a quantitative histological classification system for the assessment of intestinal mucosal biopsies. Methods: We performed a computerassisted quantitative histological assessment of digital images of duodenal biopsies from 137 controls and 124 patients with celiac disease (CeD) (derivation cohort). From the receiver-operating curve analysis, followed by multivariate and logistic regression analyses, we identified parameters for differentiating control biopsies from those of the patients with CeD. We repeated the quantitative histological analysis in a validation cohort (105 controls and 120 patients with CeD). On the basis of the results, we propose a quantitative histological classification system. The new classification was compared with the existing histological classifications for interobserver and intraobserver agreements by a group of qualified pathologists. Results: Among the histological parameters, intraepithelial lymphocyte count of $\geq 25 / 100$ epithelial cells, adjusted villous height fold change of $\leq 0.7$, and crypt depth-to-villous height ratio of $\geq 0.5$ showed good discriminative power between the mucosal biopsies from the patients with CeD and those from the controls, with $90.3 \%$ sensitivity, $93.5 \%$ specificity, and $96.2 \%$ area under the curve. Among the existing histological classifications, our quantitative histological classification showed the highest intraobserver $(69.7 \%-$ 85.03\%) and interobserver (24.6\%-71.5\%) agreements. Conclusions: Quantitative assessment increases the reliability of the histological assessment of mucosal biopsies in patients with CeD. Such a classification system may be used for clinical trials in patients with CeD. (Intest Res 2019;17:387-397)

Key Words: Celiac disease; Intestine, small; Morphometry; Image analysis; Histology, quantitative

Received December 18, 2018. Revised March 8, 2019.

Accepted March 13, 2019.

Correspondence to Prasenjit Das, Department of Pathology, All India Institute of Medical Sciences, Ansari Nagar, New Delhi 110029, India.

Tel: +91-11-26594979, Fax:+91-11-26588663,E-mail: prasenaiims@gmail. com

Co-Correspondence to Govind K Makharia, Department of

Gastroenterology and Human Nutritions, All India Institute of Medical Sciences, Ansari Nagar, New Delhi 110029, India. Tel: +91-11-26564425,

Fax:+91-11-26588663, E-mail: govindmakharia@gmail.com

ORCID Prasenjit Das (https://orcid.org/0000-0002-2420-8573), Govind K Makharia (https://orcid.org/0000-0002-2474-2194)

${ }^{5}$ Current affiliation: Celiac Disease Research Laboratory, Department of Pediatrics, Università Politecnica delle Marche, Ancona, Italy.

\section{INTRODUCTION}

The existing histological classification systems for assessment of duodenal mucosal changes in celiac disease (CeD) include original Marsh classification, modified Marsh classification, ${ }^{1,2}$ Corrazza and Villanacci classification (2005), and Ensari (2010) classification. Because of the qualitative nature of the parameters included in all the above classification system, there are low interobserver agreements amongst pathologists in most of the parameters except for the intraepithelial lymphocyte (IEL) count. ${ }^{3-6}$ The lowest interobserver agreement 
has been observed in differentiating the modified Marsh grades $3 \mathrm{a}$ and $3 \mathrm{~b}$ with these classification systems. ${ }^{5}$ Apart from the visual microscopic assessment techniques, quantitative histological assessment (Q-histology) of intestinal mucosa has gained acceptance in the recent past. ${ }^{7-10}$ Although the Qhistology was introduced in the 1970s, their use, however, remained limited to the research settings only. ${ }^{11}$ Even with this limited use, the Q-histology assessment technique evolved over a period of time and became more straightforward from older methods of measuring the mucosal surface to volume ratio, mucosal index (mucosal height to length of muscularis mucosa) assessment ${ }^{12}$ and computer card based drawing technique ${ }^{8}$ to a more simplified recent protocol of using the villous height $(\mathrm{Vh})$ to crypt depth $(\mathrm{Cd})$ ratio. ${ }^{9}$ However, despite its acceptance and simplification, Q-histology has yet not become the standard for clinical care. One of the reasons being a lack of a well defined Q-histological classification system based on quantifiable histology parameters. ${ }^{13}$

The primary objective of this study was to develop an objective Q-histological classification system for assessing the mucosal changes in the duodenal biopsies from patients with CeD.

\section{METHODS}

\section{Ethics Statement}

All study procedures were approved by the Institutional Ethical Committee (IEC/NP-312/2012 \& RP-07/2012, dated October 10,2012) and performed in the study were by the ethical standards of the institutional research committee and with the 1964 Helsinki declaration and its later amendments or comparable ethical standards. Informed consent was obtained from the subjects included in this study.

\section{Derivation Cohort}

Duodenal biopsies from 137 controls including those with functional dyspepsia $(n=50)$, subjects undergoing endoscopic retrograde cholangiopancreatography for common bile duct stone(s), as well as carriers of HBV infection were included. All of them underwent appropriate workup for the exclusion of any organic disease. All of them had negative IgA-anti-tissue transglutaminase antibody (anti-tTG Ab) titers and had normal endoscopic findings. Duodenal biopsies from 124 adult patients with CeD diagnosed as per the European Society of Pediatric Gastroenterology, Hepatology and Nutrition 2012 criteria, were also included. ${ }^{14}$ During the execution of this work as anti-tTG Ab ELISA kits from different manufacturers were used; for uniformity, we derived anti-tTG antibody fold rise value, as the (tTG value in a patient $\div$ normal cutoff value for that particular ELISA kit). During endoscopic examination, 4 to 6 mucosal biopsy fragments were obtained from the post-ampulla part of the duodenum.

\section{Identifcation of Quantitative Histological Parameters for Diferentiation between Duodenal Biopsies from Controls and Patients with CeD}

Digitized images of duodenal biopsies were analyzed by a pre-calibrated (in micrometer scale) computer assisted image analysis (CIA) technique based software supplied by the Media Cybernetics, version 6.1 (Rockville, MD, USA). The following parameters were measured in the oriented part of biopsy fragments: Vh, Cd, epithelial cell height (Eh) at the villous tip, villous area and number of IELs/100 epithelial cells (ECs) at the villous tip. The Q-histological assessment was performed on an average of 4 mucosal biopsy fragments from each subject, including at least 5 to 15 villi (depending on the biopsy orientation). CD3 staining of the IELs was done for uniformity by using the rabbit anti-human CD3 antibody (Spring Biosciences, Pleasanton, CA, US; 1:200) and was detected by a polymer-based secondary detection kit (DAKO, Carpinteria, CA, USA). The stained IELs were counted by using the manual tagging tool of the software and expressed as the number of IELs/100 ECs, after counting at least 2,000 ECs in the upper one-third of villi. The Vh and Cd were measured by using the distance measuring tool and the average $\mathrm{Cd}$ to $\mathrm{Vh}$ ratio was calculated. The histological parameters which can differentiate a biopsy from patients with $\mathrm{CeD}$ and controls were identified by receiver operating curve (ROC) analysis (Table 1) followed by multivariate and logistic regression analyses (Figs. 1, 2). Principal investigators (P.D. and A.S.) performed the CIAbased analyses in the derivation cohort in a blinded manner.

\section{Reprodicibility Assessment of the Quantitative Histological Parameters}

We then recruited a new set of validation cohort including biopsies from 120 patients with $\mathrm{CeD}$ and 105 controls. The Qhistological parameters found relevant in the derivation cohort were assessed in the validation cohort (Table 2).

\section{Formation of a New Quantitative Histological Classification}

A new Q-histological classification system for assessment of duodenal biopsies was derived based on the parameters which 


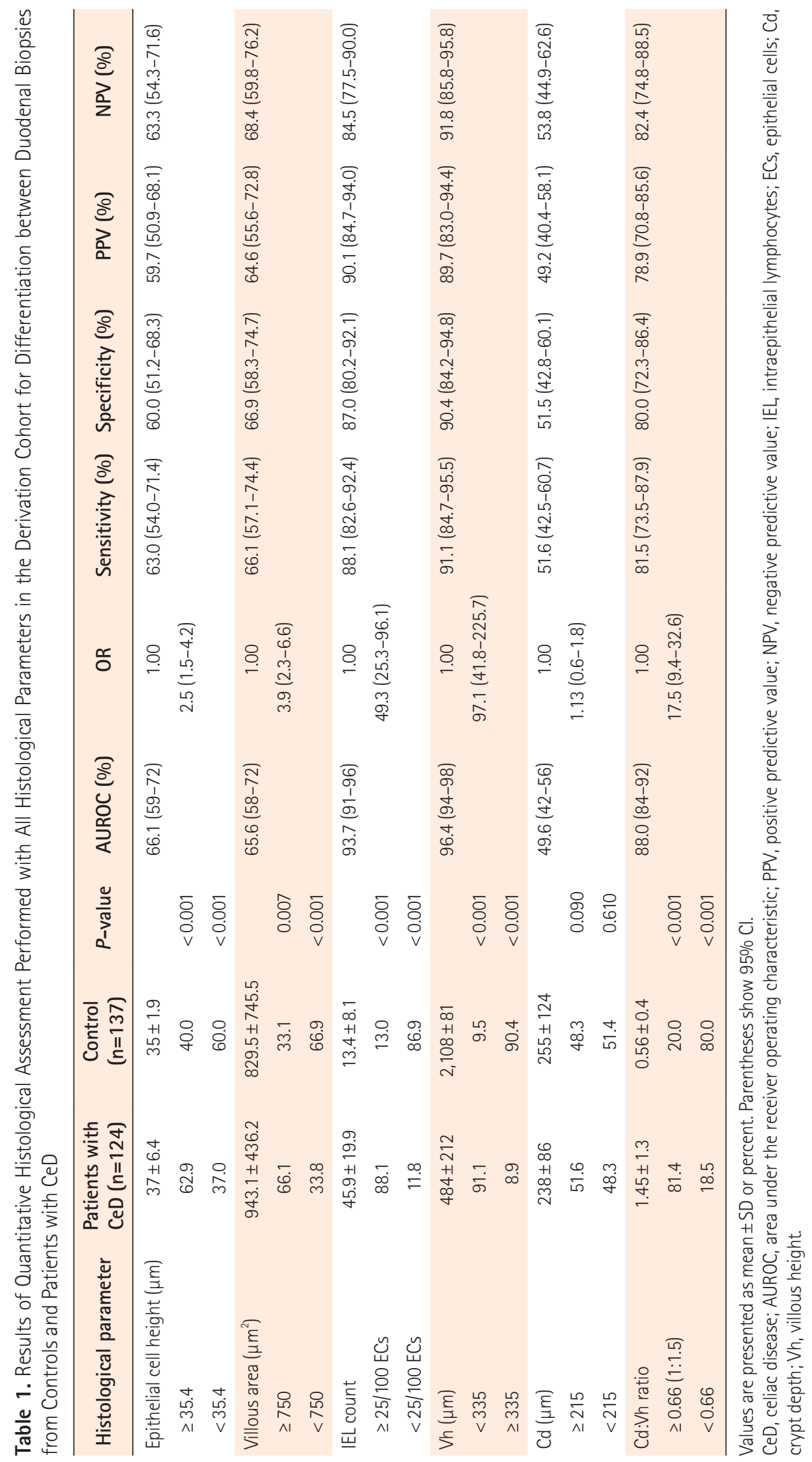




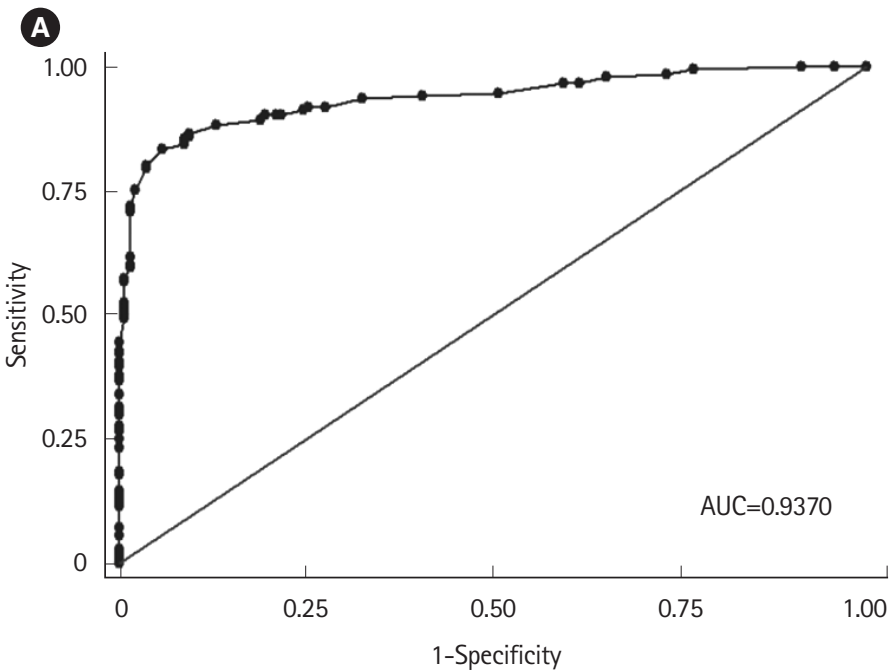

C

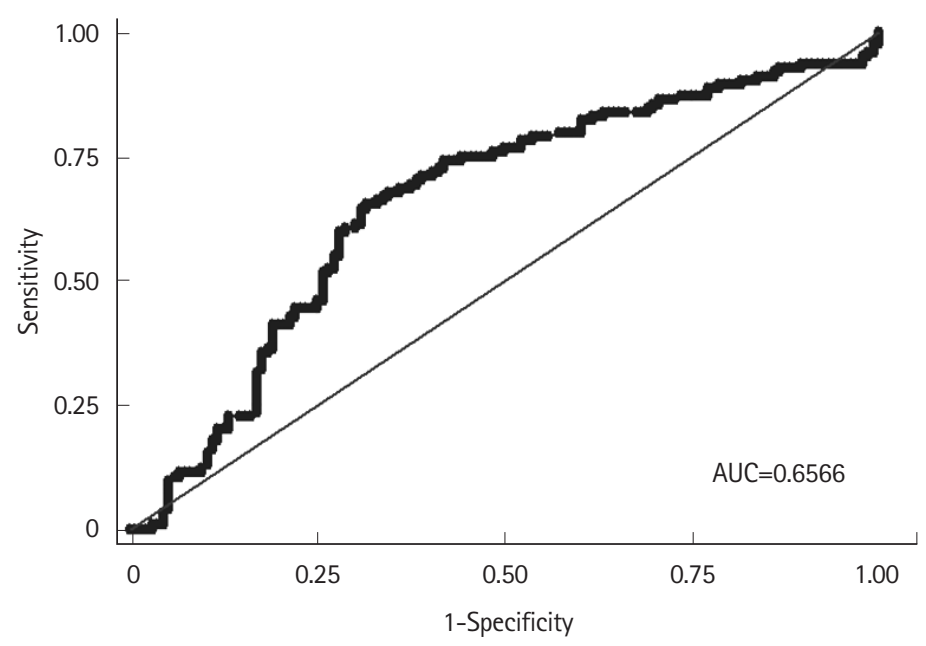

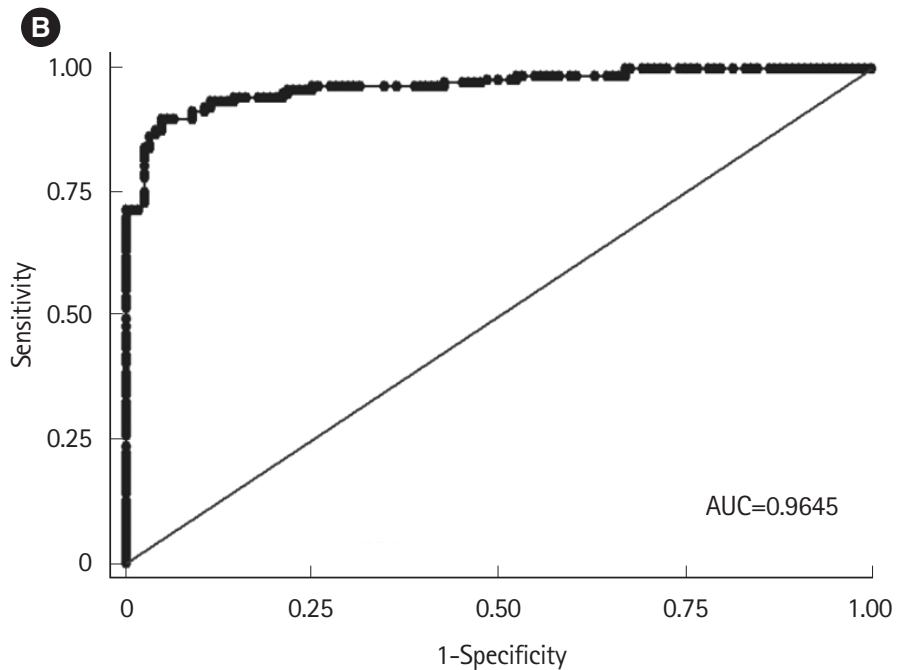

(D)

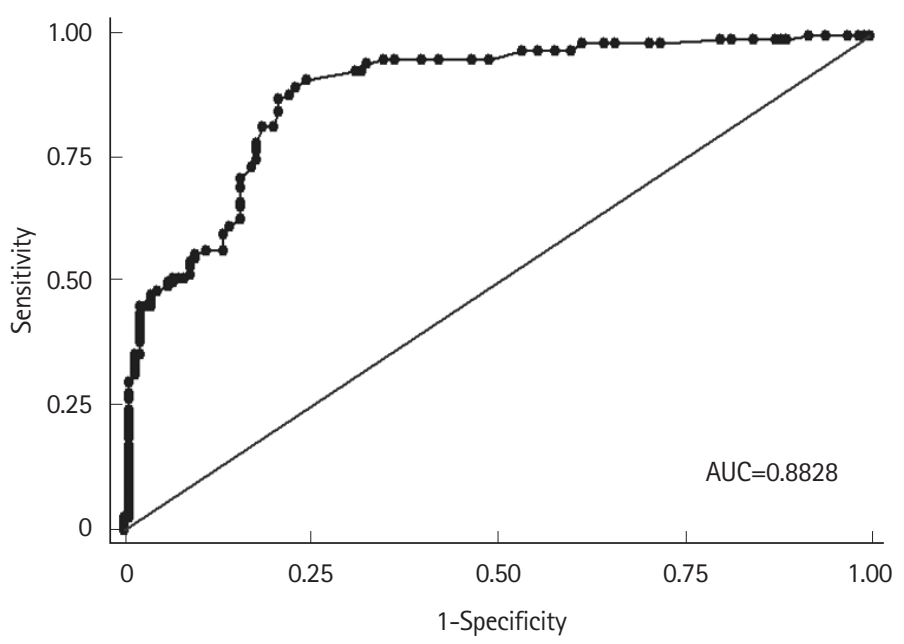

Fig. 1. (A) Showing receiver operating characteristic (ROC) considering pathological cutoff of the IEL count $\geq 25 / 100$ ECs for differentiating duodenal biopsies from patients with celiac disease and controls, with area under the ROC (AUC) of 93.7\% (95\% Cl, 91.1\%-96.1\%). (B) ROC considering pathological cutoff of $\mathrm{Vh}<335 \mu \mathrm{m}$, with AUC 96.4\% (95\% Cl, 94.3\%-98.5\%). (C) ROC considering the pathological cutoff for Cd:Vh ratio $\geq 0.66$, with AUC 88.2\% (95\% Cl, 84.1\%-92.3\%). (D) ROC considering villous area cutoff at $\geq 750 \mu m^{2}$, with AUC 65.6\% (95\% Cl, 58.8\%-72.4\%). IEL, intraepithelial lymphocytes; ECs, epithelial cells; Vh, villous height; Cd, crypt depth.

were found significant in both derivation and validation cohorts in differentiating mucosal biopsies from patients with CeD and controls (Table 3).

\section{Validation of the New Quantitative Histological Classification System}

The diagnostic sensitivity and specificity of proposed Q-histology classification were examined both in the derivation and validation cohorts separately, with and without using the antitTG antibody titers.

Intraobserver and interobserver validation: 4 qualified histopathologists (G.P.G., M.R., G.K., and A.N.), who were not in- volved in the derivation process, participated in the validation process in a blinded manner. A set of randomly selected 50 digitized histological slides of duodenal biopsies, including both controls and patients with $\mathrm{CeD}$ were handed over for Qhistological reporting using the CIA-based software. The participants were allotted different time slots on a single desktop computer to exclude technical bias. Each observer performed quantitative analysis twice, keeping a gap of 2 weeks in-between. Between the 2 cycles, the chronology of the digitized slides was altered. They were also asked to perform light microscopic grading of biopsies separately using the modified Marsh Oberhuber classification, original Marsh classification, 

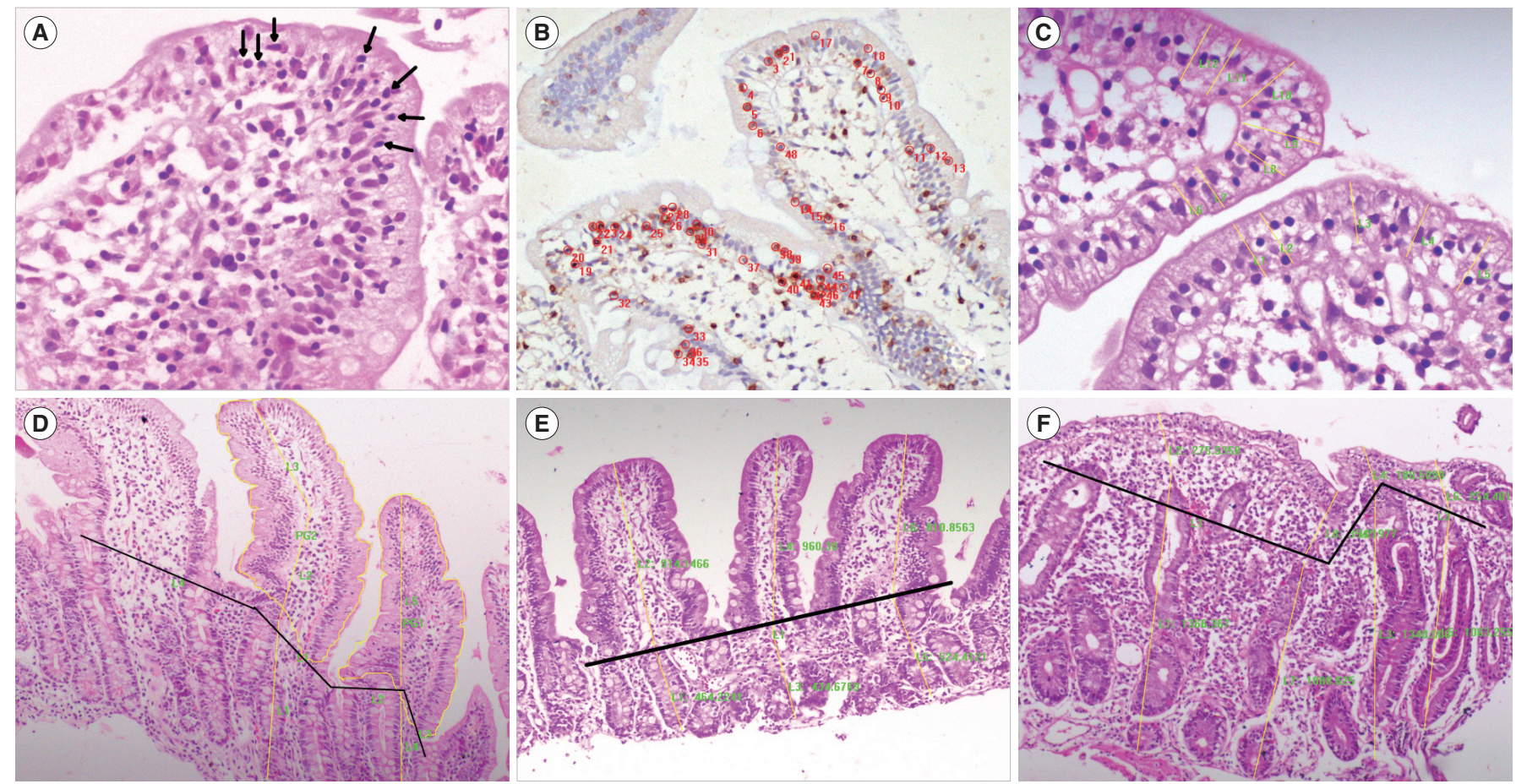

Fig. 2. (A) Photomicrograph shows a villous tip with increased intraepithelial lymphocytes (IELs; arrows) $(H \& E, \times 200)$. (B) The method of manual tagging of anti-CD3 positive IELs by using the computer assisted image analysis-based software mentioned (immunostaining, $\times 100$ ). (C) The method of measuring the mucosal epithelial heights at the villous tips ( $\mathrm{H} \& \mathrm{E}, \times 400)$. (D) Method of measuring the $\mathrm{Cd}: \mathrm{Vh}$ and the villous areas $(H \& E, \times 10)$. (E) A duodenal biopsy with increased IELs and $C d: V h>0.5$, hence, categorized as type 2 changes $(H \& E$, $\times 100$ ). (F) IEL count $\geq 25 / 100 E C s, V h$ fold change $\leq 0.7$ and $\mathrm{Cd}: V h$ ratio $\geq 0.5$, hence, categorized as type 3 changes (H\&E, $\times 100$ ). The black lines in the D-F are representing the imaginary shoulder of these biopsy fragments. $\mathrm{Cd}$, crypt depth; Vh, villous height; ECs, epithelial cells.

Corrazza and Villanacci classification, and Ensari classifications in both the occasions. The interobserver and intraobserver agreements among the observers were estimated and were compared with those with the existing light microscopic classifications.

\section{Statistical Analysis}

A Stata 11 software (StataCorp LP, College Station, TX, USA) was used. The mean \pm SD of all parameters was calculated separately. For identifying histological parameters with good discriminative power, 2 -sample $t$-test with equal variances was used, followed by 2-sample Wilcoxon signed-rank sum (Mann-Whitney) test. Diagnostic sensitivity and specificities of each parameter were calculated by ROC analysis, followed by univariate and multivariate analyses. Logistic regression analysis was performed with the calculation of OR and 95\% CIs for each parameter. The intraobserver and interobserver variations were analyzed using McNemar's test and weighted kappa analyses. A $P$-value $<0.05$ was taken as statistically significant.

\section{RESULTS}

\section{Characteristics of Subjects Included in the Derivation Cohort}

In the derivation cohort, duodenal biopsies from 261 subjects including 137 adult controls (mean age $32 \pm 10.9$ years, males 108 ) and 124 adult patients with $\mathrm{CeD}$ (mean age $26.5 \pm 13.5$ years, males 93) were included. The anti-tTG antibody fold in the control group and patients with $\mathrm{CeD}$ were $0.4 \pm 0.6$ and $8.5 \pm 8$, respectively.

\section{Quantifiable Histological Parameters Assessed in the Derivation Cohort 1) IEL Counting}

The IEL count in duodenal biopsies from patients with CeD was higher than that in controls $(45.9 \pm 19.9$ vs. $13.4 \pm 8.1, P<$ 0.001 ). IEL count $\geq 25 / 100$ ECs was found to optimally differentiate between biopsies from patients with $\mathrm{CeD}$ and control with $88 \%$ sensitivity and $87 \%$ specificity (AUC, $93.7 \%$; $95 \%$ CI, 91\%-96\%) (Table 1, Fig. 1). When the cutoff of IEL increased 
Table 3. The Proposed Quantitative Histological Classification for Assessment of Duodenal Mucosal Biopsies

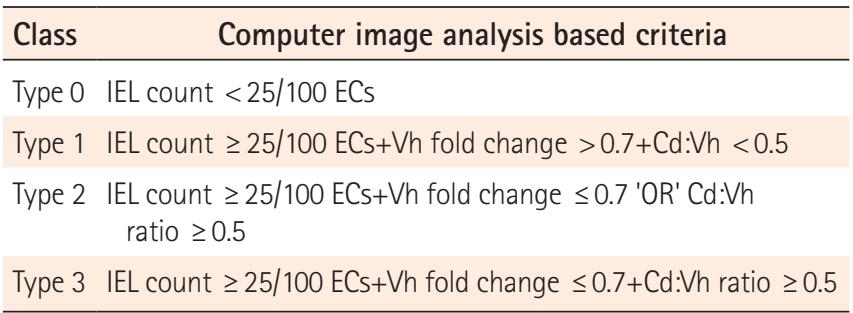

IEL, intraepithelial lymphocytes; EC, epithelial cells; Cd, crypt depth; Vh, villous height.

Notes: (1) Villous height fold change: calculated by dividing the villous height in a case $\div$ mean villous height in the control population. (2) Type 10 histology refers to-early enteropathy changes; type 2-intermediate enteropathy changes; type 3-advanced enteropathy changes. (3) A duodenal biopsy showing IEL count $<25 / 100$ ECs irrespective of normal $\mathrm{Vh}$ fold change and $\mathrm{Cd}$ to $\mathrm{Vh}$ ratio or any abnormality of the latter 2 histological parameters should be assigned 0-histology type 0 .

to $\geq 30 / 100$ ECs (as per the Marsh classification), the sensitivity reduced to $83 \%$, and specificity increased to $94 \%$. When the IEL cutoff was considered as $\geq 40 / 100$ ECs, the sensitivity dropped to only $56 \%$, but specificity increased to $99 \%$. The odds of a suspected case being CeD was $49.3(P<0.001)$ if the IEL count was $\geq 25 \mathrm{IEL} / 100 \mathrm{ECs}$ (Table 1, Fig. 1).

\section{2) Villous Height}

Vh was significantly lower in patients with $\mathrm{CeD}$, than in control biopsies $(484 \pm 212 \mu \mathrm{m}[170-1,520 \mu \mathrm{m}]$ vs. $2,108 \pm 81 \mu \mathrm{m}$ [366-3,880 $\mu \mathrm{m}], P<0.001)$. As determined by the ROC analysis, Vh cutoff $<335 \mu \mathrm{m}$ was found to have good discriminative power between the duodenal biopsies of patients with $\mathrm{CeD}$ and controls (sensitivity and specificity of $91 \%$ each [AUC 96.4\%]) (Fig. 1). The odds of a suspected case to have CeD was 97.1 (95\% CI, 41.8-225.7; $P<0.001)$, if Vh was $<335 \mu \mathrm{m}$ (Table 1).

\section{3) Crypt Depth}

There was no significant difference in the $\mathrm{Cd}$ in the mucosal biopsies obtained from controls and patients with $\mathrm{CeD}(255 \pm$ $124 \mu \mathrm{m}$ [range, $108-865 \mu \mathrm{m}$ ] vs. $238 \pm 86 \mu \mathrm{m}$ [range, $75-490 \mu \mathrm{m}$ ], $P=0.09)$ (Table 1).

\section{4) Cd to Vh Ratio}

The mean Cd to Vh ratio in controls was $0.56 \pm 0.45$ (range, $0.25-5.08$ ) in comparison to $1.45 \pm 1.3$ (range, $0.32-12.1$ ) in patients with $\mathrm{CeD}(P<0.001)$. Using the $\mathrm{Cd}$ :Vh ratio cutoff $>0.5$ (1:2), the biopsies from controls and patients were classified 
correctly with a sensitivity of $95.1 \%$ and specificity of $53.3 \%$. A Cd:Vh ratio cutoff $\geq 0.66$ (1:1.5) identified from the ROC analysis showed the highest sensitivity of $81 \%$ and specificity of $80 \%$ (AUC 88\%) (Fig. 1). As the lowest estimated Cd:Vh in the control group of biopsies was 0.25 , we then examined the discriminative power of $\mathrm{Cd}: \mathrm{Vh}>0.25$ (1:4); while the sensitivity reached to $100 \%$, specificity became nil. Our next objective was to examine the discriminative power of $\mathrm{Cd}: \mathrm{Vh}>0.33$ (1:3), as was described in the Marsh classification. With this cutoff, biopsies from patients with $\mathrm{CeD}$ and controls were classified with $99 \%$ sensitivity, but specificity was only $11 \%$. Hence, the highest discriminative sensitivity and specificity was noticed when the pathological cutoff of Cd:Vh considered $\geq 0.5$ (1:2) (Fig. 1).

\section{5) Surface Eh}

Mucosal Eh was $37 \pm 6.4 \mu \mathrm{m}$ (range, $18-58 \mu \mathrm{m}$ ) in biopsies from patients with $\mathrm{CeD}$ and $35 \pm 1.9 \mu \mathrm{m}$ (range, $17-177 \mu \mathrm{m}$ ) in controls $(P<0.001)$. On ROC analysis, Eh of $\geq 35.4 \mu \mathrm{m}$ was found to have a low discriminative power in differentiating biopsies from controls and that of patients with $\mathrm{CeD}$ (sensitivity, 63\%; specificity, 60\%; AUC, 66.1\% [95\% CI, 59\%-72\%]; OR, 2.55) (Table 1).

\section{6) Villous Area}

Duodenal biopsies from patients with CeD were found to have short and stubby villi with a higher overall villous area $\left(943.15 \pm 436.2 \mu \mathrm{m}^{2}\right)$, in comparison to the biopsies from controls $\left(829.5 \pm 745.5 \mu \mathrm{m}^{2}\right)(P=0.007)$. Villous area $\geq 750 \mu \mathrm{m}^{2}$ was found to discriminate biopsies from patients with $\mathrm{CeD}$ and controls with a sensitivity and specificity of $66 \%$ each, AUC $65.6 \%$, and odds of a suspected case having CeD was $3.94(P<0.001)$ (Table 1, Fig. 1).

\section{Assessment of Quantifiable Histological Parameters in a Validation Cohort}

The CIA-based Q-histological analyses were repeated in a validation cohort including duodenal biopsies from 225 subjects including 105 controls (mean age, $33.8 \pm 9.6$ years) and 120 patients with $\mathrm{CeD}$ (mean age, $28.5 \pm 12$ years) to re-check the reproducibility of the histological parameters (Tables 1,2). While, the pathological cutoffs for the Eh and villous area differed widely (cutoff of Eh and cutoff of villous area in derivation cohort were $\geq 35.4 \mu \mathrm{m}$ and $\geq 750 \mu \mathrm{m}^{2}$; cutoff of Eh and cutoff of villous area in validation cohort were $\geq 29 \mu \mathrm{m}$ and $<1,330 \mathrm{~m}^{2}$, respectively), rest of the histological parameters, as Vh, Cd, Cd:Vd correlated well between these cohorts (Tables 1,2).

\section{Univariate and Multivariate Analysis of the Q-Histological Parameters}

Based on univariate analysis of the results of the derivation cohort parameters such as IEL count $\geq 25 / 100 \mathrm{ECs}$, Vh $<335$ $\mu \mathrm{m}$, Cd:Vh ratio $\geq 0.66$, villous area $\geq 750 \mu \mathrm{m}^{2}$ and mucosal Eh of $\geq 35.4 \mu \mathrm{m}$ were found to be significant in the differentiation of duodenal mucosal biopsies from controls and patients with CeD with sensitivity and specificity of $62.9 \%$ and $90.4 \%$, respectively (Table 1). Of these, only 3 parameters, namely Vh $<335 \mu \mathrm{m}$, IEL count $\geq 25 / 100 \mathrm{ECs}$, and Cd:Vh ratio $\geq 0.66$, were found statistically significant on multivariable analysis. Finally, these 3 histological parameters were chosen for inclusion in the Q-histology based classification.

\section{Logistic Regression Analysis of the Q-Histological Parameters}

Based on the coefficients of logistic regression analysis, a histological scoring system was formulated to check their utility for differentiationg duodenal biopsies from patients with $\mathrm{CeD}$ and controls. The scoring system was as follows:

(IEL count $\geq 25 / 100 \mathrm{ECs} \times 3.9)+(\mathrm{Vh}<335 \mu \mathrm{m} \times 4.8)+(\mathrm{Cd}: \mathrm{Vh}$ ratio $\geq 0.66 \times 2.9$ )

Total score varied from 0 to 11 . This scoring system was then re-applied on the raw data of derivation cohort including duodenal biopsies from 261 subjects. A score of $\geq 6$, was found to discriminate the duodenal biopsies from patients with $\mathrm{CeD}$ and controls with a sensitivity of $90.3 \%$ (83.7\%-94.9\%), specificity of 93.5\% (87.7\%-97.2\%), positive predictive value (PPV) 93.3\% (87.3\%-97.1\%), negative predictive value $90.6 \%$ (84.2\%95.1\%) and AUC 96.2\%. In controls, the score was $<6(P<$ 0.001 ). These 3 Q-histological parameters together showed an area under ROC of $97.4 \%$ for discriminating between duodenal mucosal biopsies from patients with $\mathrm{CeD}$ and controls.

\section{Modification of Histological Parameters to Exclude Technical Confounding Factors}

While measuring the length between 2 points with the CIAbased software takes into account pixel density per inch, the pixel resolution of the camera sensor or the computer screen used for image analyses can alter the outcome. Hence, we decided to use a single camera for digitization and one computer terminal for CIA-based analysis. However, as these factors can vary among different set-ups, we modified the absolute cutoff 
value for Vh to Vh fold change by dividing the mean Vh in duodenal biopsies from patients by the average Vh in control biopsies. The IEL counting was based on manual tagging tool of the software, and the Cd:Vh ratio required no further adjustment. Hence, finally, the following modified histological parameters were incorporated in the proposed Q-histological classification system: IEL count $\geq 25 / 100 \mathrm{ECs}$, adjusted Vh fold change $\leq 0.7$, Cd:Vh ratio $\geq 0.5$.

\section{Proposition of a Q-Histological Classification System}

Based on the above mentioned adjusted parameters, we propose a new Q-histological classification system for classifying the changes in duodenal biopsies. We classified the biopsies to be normal (type 0 ) if IELs count is $<25 / 100$ ECs. We classified abnormalities in the biopsies as follows: type 1 or early enteropathic changes: IEL count $\geq 25 / 100$ ECs with normal Vh fold change $>0.7$ and Cd:Vh ratio $<0.5$; type 2 or intermediate enteropathic changes: IEL count $\geq 25 / 100$ ECs along with abnormality of any one of the other $2 \mathrm{Q}$-histology parameters, such as the Vh fold change $\leq 0.7$ 'OR' the Cd:Vh ratio $\geq 0.5$; type 3 or advanced enteropathic changes: IEL count $\geq 25 / 100$ ECs along with Vh fold change $\leq 0.7$, as well as the Cd:Vh ratio $\geq 0.5$ (Table 3, Fig. 2).

\section{Validation of the Newly Proposed Q-histological Classification System}

The newly proposed Q-histological classification was reapplied on the raw data files of the derivation and validation cohorts separately. Based on the defined criteria, of 323 duodenal biopsies in the derivation cohort, 142 (43.9\%) were classified as Q-histology type 0 (including 120 controls and 22 from patients with CeD), 11 (3.4\%) as Q-histology type 1 (including 7 controls and 4 from patients with CeD), 131 (40.5\%) as Qhistology type 2 (including 10 controls and 121 from patients with $\mathrm{CeD}$ ) and 39 (12.07\%) as Q-histology type 3 (including 1 control and 38 from patients with $\mathrm{CeD}$ ). The sensitivity and specificities of the Q-histology type 1, type 2 and type 3 changes were as follows: type 1: $15.4 \%$ and $94.5 \%$, which when combined with the anti-tTG Ab data became $16.7 \%$ and $99.2 \%$; type $2: 84.6 \%$ and $92.3 \%$, which when combined with tTG fold rise value became $82.1 \%$ and $99.2 \%$; type $3: 63.3 \%$ and $99.2 \%$, which when combined with tTG value became $64.3 \%$ and $100 \%$, respectively. When both the serum tTG titre was high (both anti-tTG fold rise value $>1$ and $>10$ times above normal were considered separately) and either type 1, type 2 or type 3 Q-histological changes, none of the biopsies from patients with CeD was wrongly classified as biopsies from controls.

Of duodenal biopsies from 225 patients in the validation cohort (105 controls and $120 \mathrm{CeD}$ ), 108 biopsies (47.7\%) were classified as Q-histology type 0 (all were from controls), 6 (2.6\%) as Q-histology type 1 (all 6 were biopsies from patients with $\mathrm{CeD}$ ), 33 (14.6\%) as Q-histology type 2 (all from patients with $\mathrm{CeD}$ ), and 78 biopsies (34.9\%) as Q-histology type 3 (all from patients with $\mathrm{CeD}$ ). When both Q-histology and serum tTG titres (both anti-tTG fold rise values $>1$ and $>10$ were considered) were abnormal, none of the disease biopsies were classified as controls in the validation cohort. When both serum anti-tTG titre was normal and Q-histology was 0, none of the biopsies were classified as biopsies from CeD. In 3 biopsies from patients with $\mathrm{CeD}$, despite being serum anti-tTG titre high, Q-histology type was 0. Similarly, in 18 control duodenal biopsies with normal serum anti-tTG titer Q-histology analysis showed type 1 changes. Overall sensitivities and specificities of Q-histology type 1, type 2 and type 3 changes for diagnosing CeD were $66.7 \%$ and $100 \%$; $91.7 \%$ and $100 \%$; $96.3 \%$, and $100 \%$, respectively. Adding the tTG fold rise values did not alter these sensitivities and specificities further. Overall PPV of Q-histology type 2 changes were $92.6 \%$ and $100 \%$ in the derivation, and in validation cohorts respectively. All these findings show that Q-histological type 2 and 3 changes have good sensitivities, specificities as well as PPV in identifying abnormal intestinal biopsies, which become further specific for CeD when correlated with serum tTG values.

\section{Reproducibility Assessment of the Q-Histology Classification System}

The proposed Q-histology classification system showed the highest intraobserver agreement (69.7\%-85.0\%) and interobserver agreement $(24.6 \%-71.5 \%)$ in comparison to other existing histological classification systems (Table 4).

\section{DISCUSSION}

After analyzing duodenal biopsies from 486 subjects including both controls and patients with $\mathrm{CeD}$, we found that IEL count $\geq 25 / 100 \mathrm{ECs}$, Vh fold change $\leq 0.7$, Cd:Vh ratio $\geq 0.5$ are most reliable Q-histological parameters which can differentiate duodenal biopsies from patients with $\mathrm{CeD}$ and controls. The sensitivity, specificity, and PPV of the Q-histological classification system and better interobserver and intraobserver agreements suggest that Q-histology based classification is a better method for the assessment and documentation of duo- 
Table 4. Intraobserver and Interobserver Agreements Using Histological Classification Systems

\begin{tabular}{lcc}
\hline & $\begin{array}{c}\text { Intraobserver agreements } \\
\text { (based on weighted } \kappa \text {-value) }\end{array}$ & $\begin{array}{c}\text { Interobserver agreements } \\
\text { (based on weighted } \kappa \text {-value) }\end{array}$ \\
\hline Histological classification systems & 39.7 to 60.9 & 12.9 to 48.2 \\
$\quad$ Marsh classification ${ }^{2}$ & 46.6 to 63.7 & 28.3 to 48.7 \\
Modified Marsh $^{1}$ & 54.6 to 84.6 & 28.04 to 42.9 \\
Corazza et al. $^{5}$ & 54.5 to 64.5 & 28.04 to 42.9 \\
Ensari $^{6}$ & 69.7 to 85.0 & 24.6 to 71.5 \\
\hline Proposed quantitative histological classification & & \\
\hline
\end{tabular}

All unit of data is percentage.

denal mucosal biopsies. ${ }^{15-18}$ We typed the duodenal mucosal histological changes based on the presence or absence of Qhistological parameters, as early enteropathy changes (type 1), intermediate enteropathy changes (type 2) and advanced enteropathy changes (type 3). This typing method of duodenal histological changes was however not based on the severity of Q-histological parameters, as that does not have any impact on patient management and the changes may not follow a steady pattern in reality. In this Q-histology classification system, the type 1 change only corresponds to increased IELs at villous tips; while in type 2 and type 3 changes, there will be either change in Vh or Vh:Cd or both. This way of graded typing of the histological changes will increase the likelihood of identifying the diseases biopsies more confidently, as we have seen in this study for diagnosing CeD. ${ }^{19-25}$ Though, we performed CD3 immunostaining for highlighting the IELs (Fig 2B); in routine, it is not essential. ${ }^{25}$ An experienced pathologist can identify the IELs and nuclei of ECs differently and can count easily. The other included parameters as $\mathrm{Vh}$ and $\mathrm{Cd}: \mathrm{Vh}$ can either be measured by eye-piece reticules or with CIAbased software, more easily than previously described quantitation methods, as villous tortuosity and villous area, ${ }^{26,27}$ and spatiotemporal mucosal changes by in-vivo capsule endoscopy. ${ }^{3,8,9,28}$

The Q-histology may prove to be better than the existing qualitative classification systems because it includes quantifiable histological parameters, hence can generate reliable and comparable data among research groups from different geographical regions and its ability to provide uniformity in the interpretation. As a long time is required for visual appreciation of histological improvement on the qualitative scale, another potential application of the Q-histology based classification system is likely to be assessment of even minor changes in the histology that occur over a shorter period of time espe- cially during clinical trial of newer drugs such as as latiglutenase, zonulin inhibitors, therapeutic vaccines, etc. ${ }^{29}$ Applicability of the modified Marsh classification is not optimum for interpreting follow-up biopsies from patients with CeD on GFD, because visible changes in Vh or $\mathrm{Cd}: \mathrm{Vh}$ are slow to take place and minor changes are difficult to determine visually. Q-histology in such scenario can accurately measure any improvement or deterioration of Vh or Cd:Vh, which can be compared with baseline biopsy data. The outcome of the Q-histology assessment would depend on the exclusion of the possible confounding factors, such as the technical expertise of operators, adequacy of sampling, biopsy orientation, pixel densities of the digital camera sensor and the computer screen. ${ }^{2933}$ Also, the Q-histological assessment may be more labor intensive than the visual light microscopic analysis. While the light microscopic evaluation with use of eye-piece reticule takes around 5 minutes, the CIA-based assessment may take approximately 20 minutes for analyzing a digitized slide. We took care of the possible confounding factors and tried to justify why despite being more labor intensive Q-histology can be valuable.

While there is a merit of this study, there exits a few limitations. Recruitment of entirely healthy subjects for the derivation of normative histological characteristics was not ethically justifiable, and hence we recruited subjects with functional dyspepsia, HBV carriers and patients undergoing endoscopic retrograde cholangiopancreatography as controls.

In conclusion, this study demonstrates that the Q-histological assessment of duodenal biopsy can bring uniformity and reliability in the histological evaluation of mucosal alterations in patients with $\mathrm{CeD}$. The Q-histology classification system has a potential for its use in clinical practice and in assessing histological response over a short period as required in many ongoing clinical trials. 


\section{FINANCIAL SUPPORT}

The work was supported by the Intramural Research Grant, All India Institute of Medical Sciences, New Delhi, India (grant number: A130).

\section{CONFLICT OF INTEREST}

No potential conflict of interest relevant to this article was reported.

\section{AUTHOR CONTRIBUTION}

Das P conceived idea, received the grant, executed the study \& is the guarantor of the article. Makharia GK managed all patients and controls, conceived the idea, and performed the study. Gupta SD designed and executed the study. Ahuja V supervised the study. Gahlot GP, Singh A, Yadav R, Baloda V, Rawat R, Verma A recruited the patients, collected data and work execution. Nalwa A, Roy M, George A, Ashok S, Ramteke P, Khanna G participated as an observer. Sreenivas V statistical analyses. Approval of final manuscript: all authors.

\section{ACKNOWLEDGEMENTS}

We acknowledge the contribution of staffs of Histopathology Research Laboratory and Celiac Disease Clinic.

\section{REFERENCES}

1. Oberhuber G, Granditsch G, Vogelsang H. The histopathology of coeliac disease: time for a standardized report scheme for pathologists. Eur J Gastroenterol Hepatol 1999;11:1185-1194.

2. Marsh MN. Gluten, major histocompatibility complex, and the small intestine. A molecular and immunobiologic approach to the spectrum of gluten sensitivity ('celiac sprue'). Gastroenterology 1992;102:330-354.

3. Revised criteria for diagnosis of coeliac disease. Report of Working Group of European Society of Paediatric Gastroenterology and Nutrition. Arch Dis Child 1990;65:909-911.

4. Madan M, Piplani S, Sharma M, Bhasin TS, Manjari M, Kaur H. Celiac disease: an assessment of subjective variation and diagnostic reproducibility of the various classification systems. Glob J Med Res 2015;15(No 1-C):882.

5. Corazza GR, Villanacci V, Zambelli C, et al. Comparison of the interobserver reproducibility with different histologic criteria used in celiac disease. Clin Gastroenterol Hepatol 2007;5:838843.

6. Ensari A. Gluten-sensitive enteropathy (celiac disease): controversies in diagnosis and classification. Arch Pathol Lab Med 2010;134:826-836.

7. Cummins AG, Alexander BG, Chung A, et al. Morphometric evaluation of duodenal biopsies in celiac disease. Am J Gastroenterol 2011;106:145-150.

8. Meinhard EA, Wadbrook DG, Risdon RA. Computer card morphometry of jejunal biopsies in childhood coeliac disease. J Clin Pathol 1975;28:85-93.

9. Taavela J, Koskinen O, Huhtala H, et al. Validation of morphometric analyses of small-intestinal biopsy readouts in celiac disease. PLoS One 2013;8:e76163.

10. Kuitunen P, Kosnai I, Savilahti E. Morphometric study of the jejunal mucosa in various childhood enteropathies with special reference to intraepithelial lymphocytes. J Pediatr Gastroenterol Nutr 1982;1:525-531.

11. Ciclitira PJ, Evans DJ, Fagg NL, Lennox ES, Dowling RH. Clinical testing of gliadin fractions in coeliac patients. Clin Sci (Lond) 1984;66:357-364.

12. Tulloh EA, Baylis JM, Challacombe DN. Automated analysis of morphological change in the duodenal mucosa of children with coeliac disease. Arch Dis Child 1981;56:860-863.

13. Ghoshal UC, Gwee KA. Post-infectious IBS, tropical sprue and small intestinal bacterial overgrowth: the missing link. Nat Rev Gastroenterol Hepatol 2017;14:435-441.

14. Husby S, Koletzko S, Korponay-Szabó IR, et al. European Society for Pediatric Gastroenterology, Hepatology, and Nutrition guidelines for the diagnosis of coeliac disease. J Pediatr Gastroenterol Nutr 2012;54:136-160.

15. Adelman DC, Murray J, Wu TT, Mäki M, Green PH, Kelly CP. Measuring change in small intestinal histology in patients with celiac disease. Am J Gastroenterol 2018;113:339-347.

16. Elli L, Branchi F, Sidhu R, et al. Small bowel villous atrophy: celiac disease and beyond. Expert Rev Gastroenterol Hepatol 2017;11:125-138.

17. Singh P, Kurray L, Agnihotri A, et al. Titers of anti-tissue transglutaminase antibody correlate well with severity of villous abnormalities in celiac disease. J Clin Gastroenterol 2015;49: 212-217.

18. Rahmati A, Shakeri R, Sohrabi M, et al. Correlation of tissue transglutaminase antibody with duodenal histologic marsh grading. Middle East J Dig Dis 2014;6:131-136.

19. Hammer ST, Greenson JK. The clinical significance of duodenal lymphocytosis with normal villus architecture. Arch Pathol 
Lab Med 2013;137:1216-1219.

20. Trejdosiewicz LK. What is the role of human intestinal intraepithelial lymphocytes? Clin Exp Immunol 1993;94:395-397.

21. Veress B, Franzén L, Bodin L, Borch K. Duodenal intraepithelial lymphocyte-count revisited. Scand J Gastroenterol 2004; 39:138-144.

22. Järvinen TT, Collin P, Rasmussen M, et al. Villous tip intraepithelial lymphocytes as markers of early-stage coeliac disease. Scand J Gastroenterol 2004;39:428-433.

23. Shalimar DM, Das P, Sreenivas V, Gupta SD, Panda SK, Makharia GK. Mechanism of villous atrophy in celiac disease: role of apoptosis and epithelial regeneration. Arch Pathol Lab Med 2013;137:1262-1269.

24. Ghosal UC, Das P. Diagnosis of celiac disease. In: Makharia GK, ed. Handbook of celiac disease. 1st ed. New Delhi: Kontentworx Publications, 2015:63-85.

25. Švajdler M, Daum O, Rychlý B. Diagnosing celiac disease: role of the pathologists. Int J Celiac Dis 2014;2:70-75.

26. Rosekrans PC, Meijer CJ, Polanco I, Mearin ML, van der Wal AM, Lindeman J. Long-term morphological and immunohistochemical observations on biopsy specimens of small intestine from children with gluten-sensitive enteropathy. J Clin Pathol 1981;34:138-144.

27. Boruah D, Bhatia JK, Kamal KD, Malik A. Morphometric changes in jejunal mucosa in HIV positive patients presenting with enteropathy: an Indian study. Ann Pathol Lab Med 2017;4: A379-A387.

28. Hegenbart S, Uhl A, Vécsei A. Survey on computer aided decision support for diagnosis of celiac disease. Comput Biol Med 2015;65:348-358

29. Hagendorn E, Whitney-Miller C, Huber A, Potts SJ. Development of a tissue image analysis algorithm for celiac drug development. In: Potts SJ, Eberhard DA, Wharton KA, eds. Methods in pharmacology and toxicology. New York: Springer, 2015: $141-152$

30. Vécsei A, Amann G, Hegenbart S, Liedlgruber M, Uhl A. Automated Marsh-like classification of celiac disease in children using local texture operators. Comput Biol Med 2011;41:313325.

31. Ciaccio EJ, Tennyson CA, Lewis SK, Krishnareddy S, Bhagat G, Green PH. Distinguishing patients with celiac disease by quantitative analysis of videocapsule endoscopy images. Comput Methods Programs Biomed 2010;100:39-48.

32. Gottlieb K, Dawson J, Hussain F, Murray JA. Development of drugs for celiac disease: review of endpoints for Phase 2 and 3 trials. Gastroenterol Rep (Oxf) 2015;3:91-102.

33. Murray JA, Kelly CP, Green PH, et al. No difference between latiglutenase and placebo in reducing villous atrophy or improving symptoms in patients with symptomatic celiac disease. Gastroenterology 2017;152:787-798. 